\section{MS36-P5 Revisiting the structure and synthesis of technetium heptoxide}

Daniel Mast ${ }^{1}$, Bradley C. Childs ${ }^{1}$, Keith Lawler ${ }^{1}$, Henrik Braband $^{2}$, Frederic Poineau ${ }^{1}$, Kenneth R. Czerwinski ${ }^{1}$, Alfred P. Sattelberger ${ }^{3}$

1. Department of Chemistry, University of Nevada, Las Vegas, Las Vegas NV 89154, USA

2. Department of Chemistry, University of Zurich, Zurich, Switzerland

3. Energy Engineering and Systems Analysis Directorate, Argonne National Laboratory, Lemont, IL 60439, USA

email: mastd1@unlv.nevada.edu

There are two binary oxides of technetium which have been characterized in the solid state. Technetium dioxide, $\mathrm{TcO}_{2}$, crystallizes in a distorted rutile structure and is the starting point for many synthesis routes. Technetium heptoxides, $\mathrm{Tc}_{2} \mathrm{O}_{7}$, forms one of the few molecular binary metal oxides, the others including: $\mathrm{RuO}_{4}, \mathrm{OsO}_{4}$ and $\mathrm{Mn}_{2} \mathrm{O}_{7}$. In water, $\mathrm{Tc}_{2} \mathrm{O}_{7}$ forms the common pertechnetate anion but in air the volatile solid decomposes to produce the red compound(s). It has been suggested that the red compound could be $\mathrm{HTcO}_{4}$ or $\mathrm{Tc}_{2} \mathrm{O}_{5}$.

In the context of the ongoing investigation into the nature of the red compound the structure and synthesis of $\mathrm{Tc}_{2} \mathrm{O}_{7}$ were revisited. $\mathrm{Tc}_{2} \mathrm{O}_{7}$ was synthesized by a sealed tube reaction of $\mathrm{TcO}_{2}$ in a dry oxygen environment. Single crystal X-ray diffraction results confirmed the originally proposed structure of the molecular solid with smaller lattice parameters than originally determined. With new lattice parameters, the work of Fang et al. [CrystEngComm, 2016, 18, 328-33] was revisited and effects of modeling the Pulay stress using an equation of state fit for flexible materials such as molecular solids is demonstrated. The structural stability, electronic structure and bond characteristics of $\mathrm{Tc}_{2} \mathrm{O}_{7}$ were investigated by DFT analysis. In addition, gas chromatography-mass spectrometry of $\mathrm{Tc}_{2} \mathrm{O}_{7}$ was performed to narrow the field of potential candidates for the red compound.

Keywords: Technetium oxide, molecular solid, crystallography

\section{MS36-P6 Mechanisms of mechanochemical salt formation by in situ real time X-ray powder diffraction.}

Adam A.L. Michalchuk ${ }^{1,2,3}$, Ivan A. Tumanov ${ }^{3,4}$, Simon A.J. Kimber $^{5}$, Elena V. Boldyreva ${ }^{3,4}$, Colin R. Pulham ${ }^{1,2}$

1. EPSRC Centre for Continuous Manufacturing and Crystallisation

2. EaStChem School of Chemistry, University of Edinburgh, UK

3. Novosibirsk State University, Novosibirsk, Russia

4. Institute of Solid State Chemistry and Mechanochemistry SB RAS, Novosibirsk, Russia

5. European Synchrotron Radiation Facility (ESRF), Grenoble 38000, France

email: adam.michalchuk@ed.ac.uk

Mechanochemistry is becoming increasingly popular as a tool for multi-component crystallisation and solid-state chemistry. This increase in popularity has largely been due to the increased efficiency, yields and environmentally benign nature of these processes as compared to solution-based equivalents. While mechanochemistry does offer considerable benefits, it can also induce structural or chemical transformations that may be undesired and unexpected. Such transformations can cause serious issue for industrial and academic applications. In order to prevent unwanted transformations (and similarly, promote desired ones), a better understanding of the mechanisms that underpin mechanochemistry must be understood.

In the present work the ball milling of glycine + oxalic acid dihydrate was followed by in situ, real-time X-ray powder diffraction (XRPD). The present work suggests that both salt products - glycinium semi-oxalate and bis(glycinium) oxalate - form concomitantly. A comparison of the dynamics of this process to previous ex situ work[1] highlights mechanistic discrepancies between in situ and ex situ mechanochemical investigations. Interestingly, the quantities of the final crystallisation product could be fine-tuned by controlling particle size. This suggests that, in contrast to solution based methods, a mechanochemical reaction is driven by local reactant "concentrations" as opposed to their global "concentrations"'. This, in combination with the XRPD-derived dynamic curves, is used to provide a working model for mechanochemical multi-component crystallisation. Such work is crucial for the further development of mechanochemical technologies in academic and industrial application. It is only through obtaining a better understanding of these processes that they can ultimately be controlled.

This work was supported by Edinburgh University Global Research Scholarship, EPSRC CMAC, grant 1828 from the Russian Ministry of Science and Education and ESRF CH-4313.

[1] I Tumanov, A Achkasov, E Boldyreva, and V Boldyrev (2011) CrystEndComm, 2213

Keywords: Mechanochemistry, Multi-component crystals, mechanisms 